\title{
EL MAPUCHE HABLADO EN LONQUIMAY: FONEMAS SEGMENTALES, FONOTAXIS Y COMPARACIÓN CON OTRAS VARIEDADES*
}

\author{
Makarena Alejandra Sánchez Pérez** \\ Gastón Felipe Salamanca Gutiérrez ${ }^{* * *}$
}

\begin{abstract}
Resumen
Este artículo presenta los fonemas segmentales y respectivas realizaciones alofónicas del mapudungun hablado en Lonquimay, región de La Araucanía, Chile. Es una investigación de carácter descriptivo, realizada de acuerdo con el formato estándar del descriptivismo norteamericano. Se contó con el apoyo de doce hablantes adultos, bilingües de mapudungun-castellano. Se realizó un registro de audio y visual con apoyo externo. El instrumento utilizado fue una lista léxica ad hoc que permitió determinar los fonemas, variantes alofónicas y diferencias que se pueden establecer con el mapuche central y el pehuenche del Alto Bío-Bío. Los resultados más prominentes fueron: el estatus fonológico de las interdentales [t], [n] y [l] y el fono [y]; el predominio de las realizaciones sonoras de las consonantes fricativa labiodental e interdental; el estatus de [j] como alófono de / $\mathrm{t} /$, y la escasa presencia de interferencias atribuibles al español.
\end{abstract}

Palabras claves: Mapuche, fonología del mapuche, dialectología del mapuche, mapuche hablado en Lonquimay

\section{MAPUCHE SPOKEN IN LONQUIMAY: SEGMENTAL PHONEMES, PHONOTAXIS AND COMPARISON WITH OTHER VARIETIES}

\begin{abstract}
This paper presents the segmental phonemes and its respective allophonic realizations of the spoken Mapudungun in Lonquimay, Araucania region, Chile. It is a descriptive study, conducted in accordance with the standard format of American descriptivism. Twelve adults, bilingual speakers of Mapudungun-Spanish, were interviewed. An audio and visual record with external support was performed. The instrument used was an ad hoc lexical list which identified the phonemes, allophonic variations and differences that can be set with the central Mapuche and pehuenche of Alto Bío-Bío. The most prominent findings were: the phonological status of interdental [t] [n] and [l] and phone [y]; the predominance of voiced realizations of the labiodental and inter-
\end{abstract}

* Tesis de Magíster en Lingüística Aplicada: Análisis fonético-fonológico segmental del mapudungun hablado en Lonquimay y determinación del inventario de grafemas más adecuado para su representación.

** Mg. Makarena Alejandra Sánchez Pérez. Universidad de Concepción. makarenasanchez@udec.cl

*** Dr. en Lingüística. Universidad de Concepción. Concepción, Chile. gaston.salamanca@gmail.com 
dental fricative consonants; the status of $\left[\int\right]$ as an allophone of $/ \mathrm{t} f /$, and the limited presence of interferences attributable to Spanish.

Keywords: Mapuche, Mapuche phonology, dialectology Mapuche, spoken Mapuche in Lonquimay

Recibido: 09-09-2014

Aceptado: 23-01-2014 


\section{Introducción}

Este artículo se focaliza en el nivel fonológico de la lengua vernácula hablada por los mapuches que habitan en Lonquimay.

Si bien se han cubierto muchas zonas para el conocimiento de las variantes dialectales del mapudungun, aún quedan ciertos sectores que no lo han sido o no lo han sido de manera focalizada. Así, el objetivo del presente artículo es presentar una descripción, de acuerdo con el método distribucional, del nivel fonológico segmental del mapuche hablado en Lonquimay. Específicamente, se espera determinar los fonemas segmentales y sus respectivas realizaciones alofónicas; señalar la pauta combinatoria de cada una de las unidades fonémicas segmentales; y comparar el mapuche de Lonquimay con el mapuche central y el pehuenche del Alto Bío-Bío, con el fin de conocer el perfil específico de esta variante.

Esta es una investigación de carácter descriptivo, realizada de acuerdo con el formato estándar del descriptivismo norteamericano. En este sentido, se realizaron entrevistas en la zona de Lonquimay con el fin de recabar los datos necesarios. El instrumento utilizado fue una lista léxica ad hoc que permitió determinar los fonemas, variantes alofónicas y diferencias que se pueden establecer con el mapuche central y el pehuenche del Alto Bío-Bío.

Esta investigación constituye un aporte al conocimiento del mapudungun en la medida en que describe una variante no abordada de manera focal. Este aporte puede constituirse en un insumo valioso que provee el piso para realizar estudios de otra índole, como, por ejemplo, la adquisición de fonemas del mapudungun o apoyar propuestas para la elaboración de materiales en el proceso de enseñanza-aprendizaje de esta lengua.

\section{Objetivos de la investigación}

\section{Objetivo general}

Describir, de acuerdo con el método distribucional, el nivel fonológico segmental del mapuche hablado en Lonquimay. 


\section{Objetivos específicos}

1. Determinar los fonemas segmentales y sus respectivas realizaciones alofónicas del mapuche hablado en Lonquimay.

2. Señalar la pauta combinatoria de cada una de las unidades fonémicas segmentales.

3. Comparar el mapuche de Lonquimay con el mapuche central y con el pehuenche de Alto Bío-Bío.

\section{Marco de referencia teórico-metodológico utilizado en este estudio}

Hace ya varias décadas, el rescate y documentación de las lenguas en el mundo se ha vuelto de gran importancia para la preservación de éstas. Es por esto que, en Norteamérica, se desarrolló la llamada "lingüística descriptiva de salvataje", cuyo foco era el registro de las lenguas amenazadas con la extinción. Con figuras como Sapir, Pike, Hockett, entre muchos otros, se desarrolló un marco teórico-metodológico en el cual se definieron nociones como vocoides, contoides, fonemas, distribución, etc., las cuales se asentaron firmemente en la disciplina y forman la base para el análisis fonético-fonológico de datos recogidos para el rescate de lenguas en peligro. La mayoría de las descripciones fonológicas del mapudungun hablado en Chile ha sido realizada de acuerdo con este modelo.

Las premisas propuestas por Pike (1947) y Burquest (2009) para el análisis fonémico son las siguientes: un sonido tiende a ser modificado por los sonidos adyacentes, los sonidos de una lengua presentan tendencia a la simetría, y series características de sonidos ejercen una presión estructural en la interpretación fonémica de segmentos o series fónicas sospechosas.

Ahora, el estatus fonémico de los fonos articulatoriamente similares se determina de acuerdo a si entran en una relación de contrastante,

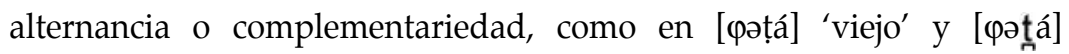
'marido', donde se observa que $[\mathrm{t}]$ y [t] contrastan y, en consecuencia, pertenecen a fonemas diferentes: / ț / y / t / (Echeverría, 1964: 29); [0omo] $\sim$ [ðomo] 'mujer', donde se observa que [ $\theta]$ y [ð] alternan libremente y, 
entonces, pertenecen al mismo fonema (Echeverría, 1964: 31); o como en [nárki] [anatún] y [pəțrien]], donde se observa que [n] y [ṇ] ocurren en contextos excluyentes y, en consecuencia, pertenecen al mismo fonema /n/ (Echeverría, 1964: 33).

Un fenómeno que se presenta con frecuencia en el mapudungun es la llamada fluctuación de fonemas; esta etiqueta se utiliza, en nuestro caso, tal como es definida en Salas (2006[1992]: 78).

En este trabajo también se incluye información con respecto a las interferencias fonológicas del español en el mapudungun hablado en la zona. El modus operandi para dar cuenta de ellas es afín con el que se presenta en los trabajos pioneros de Henríquez (2004 y 2013).

\section{Metodología de la investigación}

\subsection{Tipo de estudio}

Esta es una investigación de carácter descriptivo, realizada de acuerdo con el formato estándar del descriptivismo norteamericano, el cual es el más usado en la descripción de vernáculos chilenos. El enfoque metodológico es cualitativo; se escogió este acercamiento porque da cuenta de las propiedades importantes de los fenómenos sometidos a análisis.

\subsection{Recolección del material}

\subsubsection{Zona}

Los datos fueron recogidos en la comuna de Lonquimay, ubicada en la provincia de Malleco, Región de la Araucanía; específicamente, en las comunidades Bernardo Ñanko, sector El Naranjo, y Bernardo Nanko, sector Mallín del Treile.

A continuación, presentamos un mapa, donde se observa la zona específica donde fueron recogidos los datos.

\subsubsection{Informantes y entrevistas}

\subsubsection{Informantes}

Para esta investigación se contó con el apoyo de doce hablantes adultos, bilingües de mapudungun-castellano. Estos hablantes fueron: 


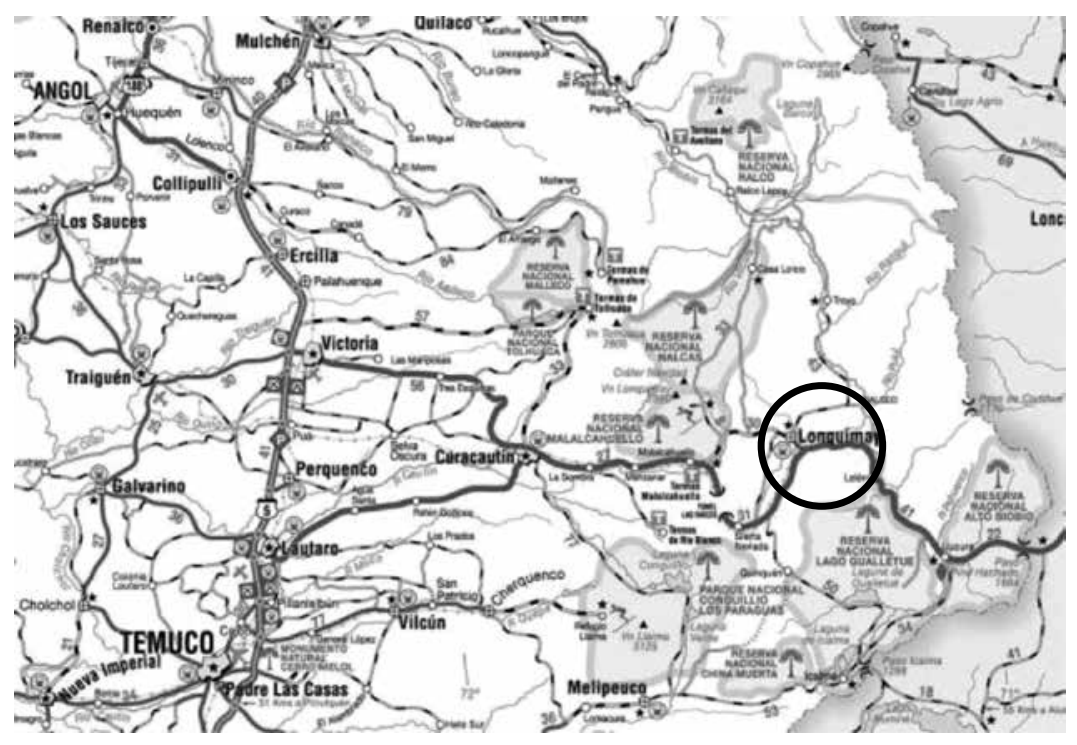

Arturo Lillo, Francisca Lillo, Flora Curical, Lilian Puel, Elena Rosas, Eugenia Nahuelcheo y Domingo Nanko, de la Comunidad Bernardo Nanko, sector El Naranjo; y Raúl Nanko, Teresa Rosas, Berta Curical, Daniel Pailla e Hilda Huilipan, de la Comunidad Bernardo Nanko, sector Mallín del Treile. Algunos de estos informantes fueron contactados por una profesora intercultural de la zona, mientras que otros fueron contactados por datos de los mismos entrevistados.

\subsubsection{Entrevistas}

Se realizaron dos viajes a la comuna de Lonquimay con el fin de poder recolectar los datos necesarios para esta investigación, por medio de la grabación de una lista léxica. Así, en el primer viaje entrevistamos a siete de nuestros colaboradores, pertenecientes a la comunidad Bernardo Nanko, sector El Naranjo; mientras que en el segundo viaje, se entrevistó al resto de los colaboradores, quienes pertenecen a la comunidad Bernardo Nanko, sector Mallín del Treile. Estas entrevistas se realizaron en la casa de los colaboradores.

\subsubsection{Registro de los datos y sesiones de transcripción}

Se realizó un registro de audio y visual con apoyo externo, con el fin de poder discriminar de forma precisa los rasgos fonético-articulatorios de los segmentos a analizar. Así, para el registro de audio se utilizó una 
grabadora TASCAM DR-100, la cual ha sido utilizada en investigaciones anteriores de estas características y sirvió para realizar las revisiones necesarias luego de las entrevistas. En cuanto al registro visual, éste se realizó con una cámara filmadora de alta definición (Sony Full HD, HDRCX240B). Ésta fue de gran utilidad, ya que permitió revisar y acceder a las imágenes las veces que fuesen necesarias para aclarar aquellas dudas que surgían al momento del análisis.

En cuanto a las transcripciones, éstas fueron de dos tipos: una en terreno, donde se puso especial atención en la presencia de los fonos interdentales, y una exhaustiva que resultó de las audiciones pasivas y deliberadas de los registros audiovisuales.

Las transcripciones fueron realizadas, en primer lugar, por la investigadora, y luego chequeadas exhaustivamente por el Profesor Guía en la Universidad de Concepción. Éstas se realizaron de acuerdo con los símbolos propuestos por el Alfabeto Fonético Internacional (AFI). Es preciso mencionar que la utilización estricta de este alfabeto para la transcripción del mapudungun sólo tiene precedentes en Henríquez y Salamanca (2012) y Sadowsky et al (2013); y la estimamos como un aporte valioso para la difusión de los resultados, tanto a nivel nacional, como internacional.

\subsubsection{Lista léxica}

Con el fin de facilitar el estudio comparativo de esta variedad del mapudungun, se empleó como base la lista léxica que aparece en Salamanca (1997). A esta lista, se agregaron algunas palabras que contienen segmentos críticos que han sido objeto focal de estudio en esta investigación.

Sin embargo, una vez en el lugar, nos percatamos de que algunos hablantes de la zona no reconocieron algunas palabras de la lista léxica. En este contexto, nos vimos en la necesidad de descartar aquellas que no fueron respondidas por los informantes y añadir algunas con el fin de equilibrar la lista. Así, la lista léxica definitiva quedó constituida por ciento tres ítems.

\subsubsection{Análisis del material}

Como señalamos en 3.2.3., se realizaron audiciones pasivas y deliberadas del material registrado en audio y video, las cuales 
permitieron transcribir todo el material. Posteriormente, se aplicaron las premisas y propuestas contenidas en el marco de referencia presentado en el punto 2 .

\section{FONEMAS SEGMENTALES DEL MAPUCHE HABLADO EN LONQUIMAY}

\subsection{Fonemas vocálicos}

A continuación, se presenta la descripción articulatoria de las realizaciones alofónicas de los fonemas vocálicos del mapuche hablado en Lonquimay, los cuales son: / a, e, i, o, u, ə/.

\subsubsection{Fonema / a /}

Este fonema se realiza como [a]: vocoide baja central no redondeada silábica. Por ejemplo, [a.'tfa.wak] 'pollo', ['ka.tfu] 'pasto', [ma.'wi.ða] 'montaña'.

\subsubsection{Fonema / e/}

Este fonema se realiza como [e]: vocoide media anterior no redondeada silábica. Por ejemplo, [e.'pu] 'dos', [wan.' 'Ien] 'estrella', [məృ. 'kje] 'harina tostada'. Existe fluctuación de fonemas entre /e/ / a/, como se observa en el siguiente ejemplo: / ne.vəð/ / na.vəð/ 'coser'.

\subsubsection{Fonema / i/}

Este fonema se realiza como [i]: vocoide alta anterior no redondeada silábica. Por ejemplo, [in.'tfe] 'yo', [kii.'ne] 'uno', ['po.ni] 'papa'. Existe fluctuación de fonemas entre $/ \mathrm{i} / \sim /$ / / y / i / / $\mathrm{u} /$, como se observa en los siguientes ejemplos: / ki.ne/ / kə.ne/, 'uno' y / ku.ni.val/ / ku.n u.val/ 'huérfano', respectivamente.

\subsubsection{Fonema / o/}

Este fonema se realiza como [o]: vocoide media posterior redondeada silábica. Por ejemplo, [o.'vi.sa] 'oveja', [to.'kii] 'hacha', [ðo.'mo] 'mujer'. Hay fluctuación de fonemas entre $/ \mathrm{o} / \sim / \mathrm{\partial} / \mathrm{y} / \mathrm{o} / \sim / \mathrm{u} /$, como se

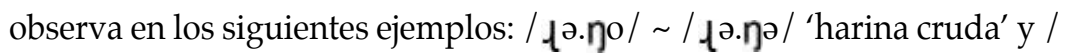
рә.ðо/ / рә.ðu/ 'ombligo', respectivamente. 


\subsubsection{Fonema / u/}

Este fonema se realiza como [u]: vocoide alta posterior redondeada silábica. Por ejemplo, [u.'le] 'mañana', [ku.'łu] 'negro', [leư.'ßßu] 'río'. Hay fluctuación de fonemas entre $/ \mathrm{u} / \sim / \mathrm{\jmath} / \mathrm{y} / \mathrm{u} / \sim / \mathrm{o} /$, como se observa en los siguientes ejemplos:

/vu.łi/ /vo.łi/ 'espalda' (esta fluctuación podría asociarse con una vitalidad de la sexta vocal en el mapudungun hablado en Lonquimay) y / ni.Ka.tun/ / ni.Ka.ton/ 'rogativa', respectivamente.

\subsubsection{Fonema / / /}

Este fonema tiene dos alófonos en distribución complementaria:

[u]: vocoide alta posterior no redondeada silábica. Ocurre en posición inicial de palabra y después de consonantes velares y retroflejas. Por ejemplo, [u.'nəm] 'pájaro', [ku.'łuuf] 'viento', ['

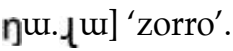

[ə]: vocoide media central no redondeada silábica. Ocurre en otros contextos. Por ejemplo, ['və.ta] 'marido' y ['an.tə] 'sol'. Hay fluctuación de fonemas entre /ə/ / e/, / / / /i/ $\mathrm{y} / \mathrm{\partial} / \sim / \mathrm{u} /$, como se observa en los siguientes ejemplos:/'n

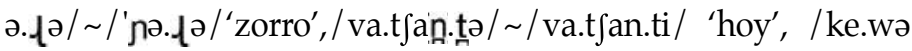
n/ /ke.wun/ 'lengua', respectivamente. Estas fluctuaciones también podrían interpretarse como influencias del español en el mapudungun de la zona.

Las siguientes tablas resumen los fonos y fonemas vocálicos de la zona.

Tabla 1: Fonos vocoides silábicos de Lonquimay

\begin{tabular}{|l|l|l|l|l|}
\cline { 2 - 5 } \multicolumn{1}{c|}{} & \multicolumn{2}{l|}{ No redondeada } & No redondeada & Redondeada \\
\hline Alta & {$[\mathrm{i}]$} & & {$[\mathrm{u}]$} & {$[\mathrm{u}]$} \\
\hline Media & {$[\mathrm{e}]$} & {$[ə]$} & & {$[\mathrm{o}]$} \\
\hline Baja & & {$[\mathrm{a}]$} & & \\
\hline
\end{tabular}


Tabla 2: Fonemas vocálicos de Lonquimay

\begin{tabular}{|l|l|l|l|}
\hline & \multicolumn{2}{|l|}{ No redondeada } & Redondeada \\
\hline Alta & li/ & & $/ \mathrm{u} /$ \\
\hline Media & le/ & $/ \mathrm{l} /$ & $/ \mathrm{o} /$ \\
\hline Baja & & $/ \mathrm{a} /$ & \\
\hline & Anterior & Central & Posterior \\
\hline
\end{tabular}

\subsection{Fonemas Consonánticos}

En el mapuche hablado en Lonquimay, los fonemas consonánticos

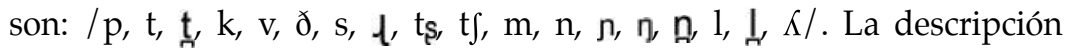
articulatoria de las realizaciones alofónicas de cada uno de ellos se presenta a continuación.

\subsubsection{Fonema / p/}

Este fonema se realiza como [p]: contoide obstruyente oclusiva bilabial sorda oral. Por ejemplo, [pa.'kał.wa] 'sapo', [tşa.'pi] 'ají'.

\subsubsection{Fonema / $\mathrm{t} /$}

Este fonema se realiza como [t]: contoide obstruyente oclusiva alveolar sorda oral. Por ejemplo, [ta.'kun] 'ropa', [ $\eta \mathrm{ji}$.Ka.'tun] 'rogativa'. Hay fluctuación de fonemas entre $/ \mathrm{t} / \sim / \mathrm{t} / \mathrm{y} / \mathrm{t} / \sim / \mathrm{t} /$, como se observa en los siguientes ejemplos: / ta.pə._a/ / tsa.pə._a/ 'muchacha soltera' y /pa.ta.ka/ / pa.ta.ka/ 'cien', respectivamente. Esta última fluctuación también podría asociarse con una vitalidad del segmento interdental.

\subsubsection{Fonema / $\mathbf{n} /$}

Este fonema se realiza como [t]: contoide obstruyente oclusiva interdental sorda oral. Por ejemplo, ['tol] 'frente', ['mə.ța] 'cuerno'.

Como se aprecia en los enunciados anteriores, [t $\mathrm{t}_{n}$ aparece en posición inicial y entre vocales, al igual que [t]; ambas consonantes, por lo mismo, se encuentran en distribución contrastante por ambiente análogo (pares submínimos). Por otra parte, según los datos obtenidos, se observa una alta consistencia en la aparición de las interdentales, tanto en las palabras elicitadas, como en los hablantes. Así, las palabras 'hoja', 'frente', 'cuerno', piojo', 'hoy' y 'sol' se realizan, en su mayoría, con el

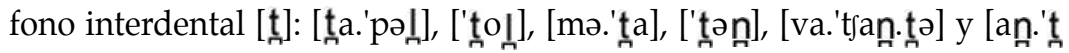


ə], respectivamente. Del mismo modo, del total de informantes (12), siete presentan consistentemente la realización interdental de este fonema. De ahí que se le asigne estatus fonémico (/ $\mathrm{t} /$ ).

Hay fluctuación de fonemas entre $/ \mathrm{t} / \sim / \mathrm{t} /$, como se observa en el siguiente ejemplo: / ta.pəI/ / ta.pəl/ 'hoja'. Esta fluctuación, tal como para el caso de / / / (/e/, /i/, / u/ ), también podría interpretarse como una influencia del español en el mapudungun de la zona.

\subsubsection{Fonema / k/}

Este fonema tiene dos realizaciones alofónicas en distribución complementaria:

[k]: contoide obstruyente oclusiva v elar sorda oral. Ocurre ante vocoide no anterior. Por ejemplo, ['ku._a] 'piedra' y [ma.'kun] 'manta'.

[kj]: contoide obstruyente oclusiva postpalatal sorda oral. Ocurre ante vocoide anterior. Por ejemplo, ['ki.ne] 'uno' y ['n u.kje] 'madre'.

\subsubsection{Fonema / ts /}

Este fonema tiene dos realizaciones alofónicas en variación libre: [ts]: contoide africada alveolar retrofleja sorda, y [t]: contoide oclusiva alveolar retrofleja sorda. Por ejemplo, [tsal.'kan] [tal.'kan] 'trueno' y [ku.'tsal] [ku.'tal] 'fuego'.

Según los datos obtenidos, la africada [t\$s] posee una mayor frecuencia, en comparación con su contraparte oclusiva [t], tal como muestra el siguiente gráfico. Esto justifica que se la haya seleccionado como la forma base del fonema $(/ \mathbf{t} /)$.

Gráfico 1: Porcentaje de realización de los segmentos [t\$s] y [t].

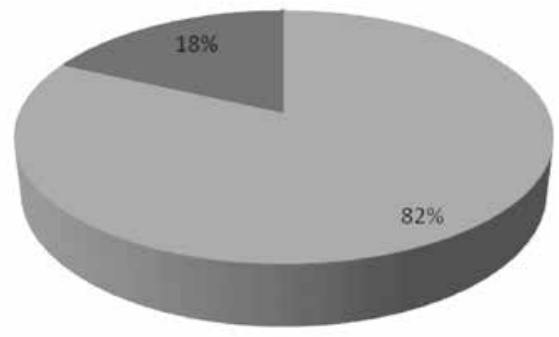


Por otra parte, se observa un caso de transferencia del español: la secuencia [tr], la cual fue articulada en una oportunidad, en la palabra 'remedio desagradable'. Así, el informante reemplaza el fono africado alveolar retroflejo sordo [ts] por [tr]; en consecuencia, donde se esperaba ['na.tsi], se articuló ['na.tri]. Cabe señalar, sin embargo, que el número de apariciones de esta secuencia es mínima, tal como se observa en el siguiente gráfico.

Gráfico 2: Porcentaje de realizaciones alofónicas y transferencias en el fonema / $\mathbf{\mathbf { S }} /$.

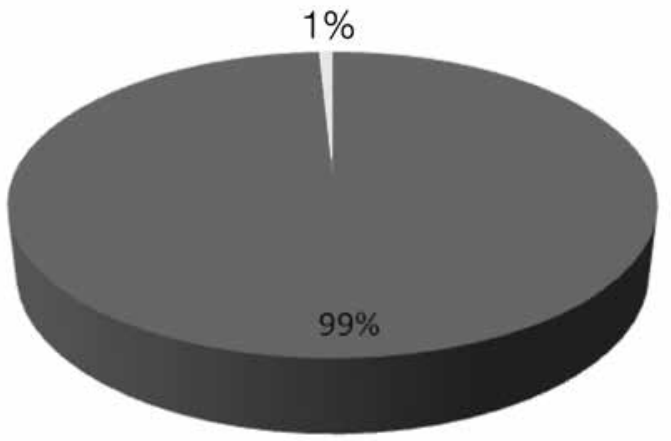

- Alófonos de /tss/ Transferencias

Hay fluctuación de fonemas entre $/ \mathrm{t} \mathbf{S} / \sim / \mathrm{t} \int /$, como se observa en el siguiente ejemplo: / pi.tsun/ / pi.tsun/ 'humo'

\subsubsection{Fonema $/ \mathrm{t} \int /$}

Este fonema tiene dos realizaciones alofónicas en variación libre: [ $\mathrm{t}$ $\left.\int\right]$ : contoide africada alveopalatal sorda oral y [s]: contoide fricativa alveopalatal sorda oral. Por ejemplo, [ka.'t fi.Ka] [ka.' $\left.\int i . j a\right]$.

Según los datos obtenidos, hubo 4 realizaciones del fono fricativo alveopalatal áfono [ $\left.\int\right]$; en todos los casos, el contexto fue la posición intervocálica (como, por ejemplo, en [ka.'Si.ja] 'trigo' y [va.' Jann.țə] ‘hoy'). De ahí que se haya seleccionado el fono africado alveopalatal sordo [t $\mathrm{t}$ ] como la forma básica del fonema. Los porcentajes precisos de aparición se muestran en el siguiente gráfico. 
Gráfico 3: Porcentaje de realización de los segmentos [t $\left.\int\right]$ y [ []].

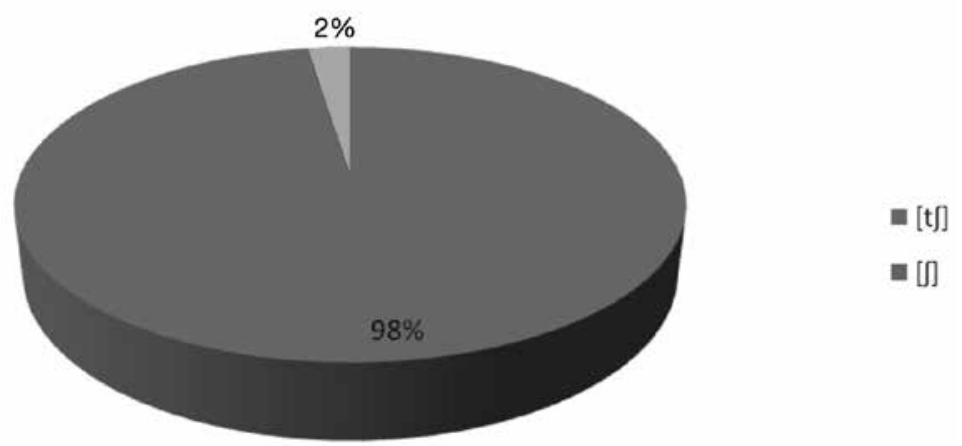

\subsubsection{Fonema / v/}

Este fonema tiene tres realizaciones alofónicas en variación libre: [v]: contoide fricativa plana labiodental sonora oral, [f]: contoide fricativa plana labiodental sorda oral, y $[\beta]$ : contoide fricativa bilabial sonora oral.

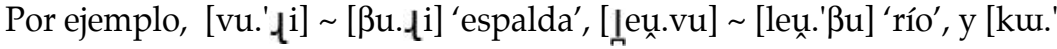
łuv] [ku.'‘uß] [ku..'łuf] 'viento'. El fono fricativo labiodental sonoro [v] alterna libremente con $[\beta]$ en las tres posiciones de referencia y en cada una ocurre con una mayor frecuencia, mientras que la realización fricativa plana labiodental sorda [f] ocurre sólo en coda silábica. De ahí que la realización sonora [v] se haya seleccionado como la forma base del fonema. Los porcentajes precisos de cada alófono se presentan en el siguiente gráfico.

Gráfico 4: Porcentaje de realización de los segmentos [v], [f] y [ß].

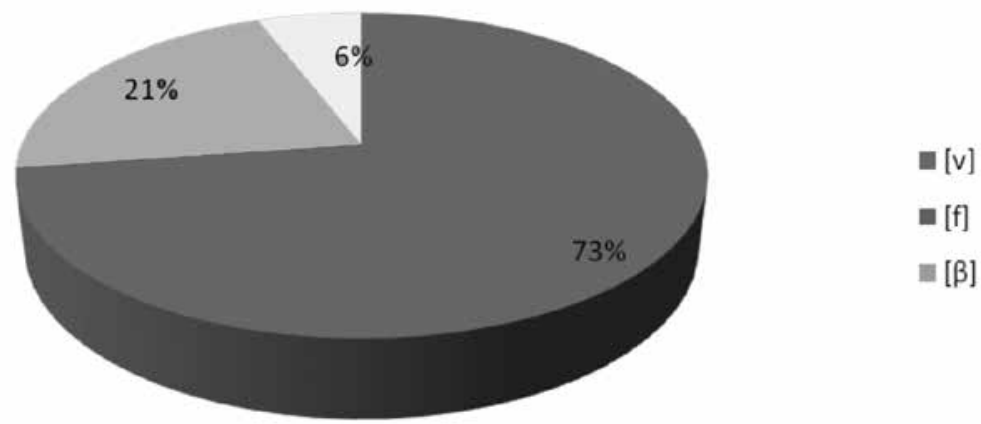


Por otra parte, cabe señalar que en algunos colaboradores se presentó la realización bilabial oclusiva [b], lo que puede ser interpretado como una transferencia desde el castellano al mapudungun. Por ejemplo, en la palabra 'espalda', algunas realizaciones fueron [bu.'zi], en vez de [vu.' zi]. Sin embargo, su ocurrencia es baja, como lo evidencia el siguiente gráfico.

Gráfico 5: Porcentaje de realizaciones alofónicas y transferencias en el fonema /v/

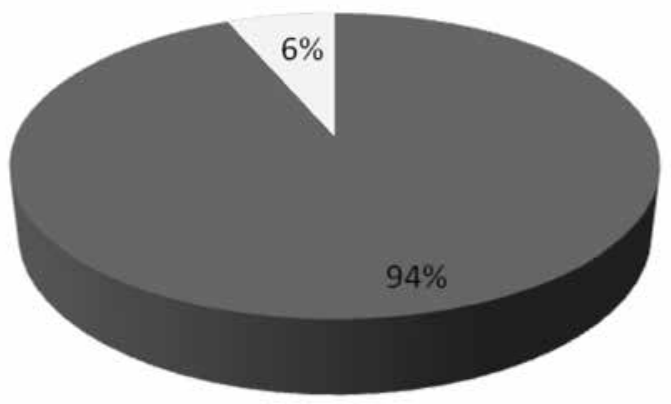

Alófonos de /v/ Transferencias

\subsubsection{Fonema /ð/}

Este fonema tiene dos realizaciones alofónicas en variación libre: [ð]: contoide fricativa plana interdental sonora y $[\theta]$ : contoide fricativa plana interdental sorda.

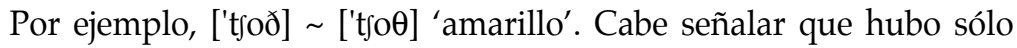
6 realizaciones del fono fricativo plano interdental sordo [ $\theta$ ] y que su ocurrencia fue exclusivamente en coda silábica (como en [pa.'nu $\theta$ ] 'suave', [tje $\theta . ' k u i]$ ] 'suegro' y ['tjo $\theta$ ] 'amarillo'). De ahí que se haya seleccionado la realización sonora [ð] como la forma básica del fonema. Los porcentajes precisos de cada alófono se presentan en el siguiente gráfico. 
Gráfico 6: Porcentaje de realización de los segmentos [ð] y $[\theta]$.

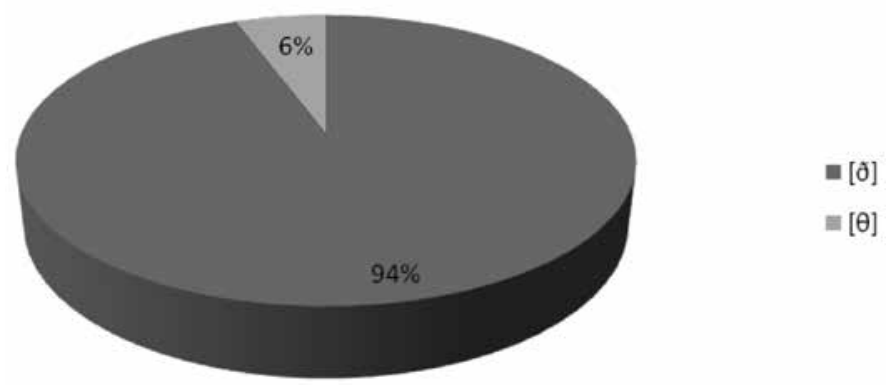

Asimismo, es preciso mencionar que en cuatro de los informantes se presentó la realización oclusiva postdental sonora [d] (específicamente, en la articulación de [do.'mo.tje] 'mujer'), lo cual puede ser interpretado como una transferencia desde el castellano al mapudungun. El porcentaje correspondiente a estas cuatro realizaciones se presenta en el siguiente gráfico.

Gráfico 7: Porcentaje de realizaciones alofónicas y transferencias en el fonema /ð/

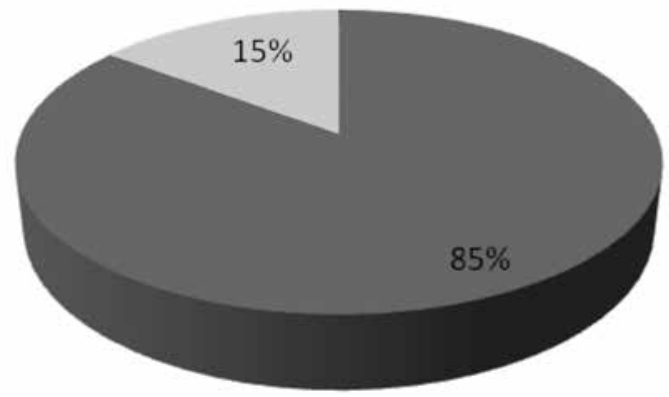

\section{- Alófonos de /ð/ = Transferencias}

Hay fluctuación de fonemas entre $/ ð / \sim / \mathrm{j} /$ como se observa en el siguiente ejemplo: / pa. nuð/ / pa.nuj/ 'suave'.

\subsubsection{Fonema / s/}

Este fonema se realiza como [s]: contoide fricativa ápicoalveolar sorda oral. Por ejemplo, ['saj.we] 'chancho' y [u.'vi.sa] 'oveja'. Se observa fluctuación de fonemas entre $/ \mathrm{s} / \sim / \mathrm{\partial} / \mathrm{y} / \mathrm{s} / \sim / \mathrm{t} /$, como se observa en 
los siguientes ejemplos: / u.vi.sa/ / u.vi.ða/ 'oveja' y / san.we/ /tja .we/ 'chancho', respectivamente.

\subsubsection{Fonema / $1 /$}

Este fonema tiene tres realizaciones alofónicas en variación libre. [^]: contoide aproximante retrofleja cacuminal sonora, [z]: contoide fricativa retrofleja cacuminal sonora y $\left[\ell_{\ell}^{\gamma}\right]$ : contoide aproximante retrofleja

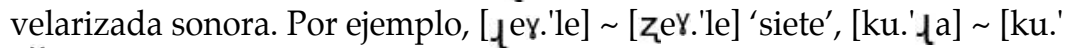
$\left.\ell^{\gamma} \mathrm{a}\right]$ 'piedra', y ['tşal] 'pus'. El fono aproximante retroflejo cacuminal sonoro [ $\downarrow]$ ocurre en las tres posiciones de referencia; [z] ocurre en el premargen silábico y entre vocales; en tanto que [ $\left.\ell^{\gamma}\right]$ ocurre sólo en el último contexto señalado. La selección de [^] como la forma base del fonema se justifica por la alta frecuencia que presenta en la lista léxica, en comparación con $[z]$ y $\left[\ell^{\gamma}\right]$. Los porcentajes precisos de cada alófono se presentan en el siguiente gráfico.

Gráfico 8: Porcentaje de realización de los segmentos [A], [Z] y [ौ] ${ }^{\gamma}$.

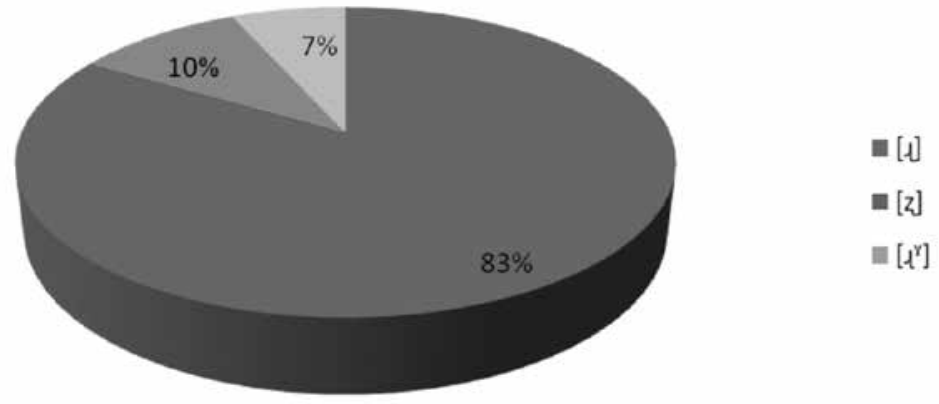

Hay fluctuación de fonemas entre / $/ / \sim /$ /, como se observa en el siguiente ejemplo: / ko.tjol/ / ko.tjoð/ 'amarillo'.

\subsubsection{Fonema $/ \mathrm{m} /$}

Este fonema se realiza como [m]: contoide nasal bilabial sonora. Por ejemplo, [man.'sun] 'buey', [ðo.'mo.tje] 'mujer' y [ku.'‘am] 'huevo'. Hay fluctuación de fonemas entre $/ \mathrm{m} / \sim / \mathrm{n} /$, como se observa en el siguiente ejemplo: /moK.vən/ / goא.vən/ 'sangre'. 


\subsubsection{Fonema /n/}

Este fonema presenta dos realizaciones alofónicas en distribución complementaria:

[n]: contoide nasal alveolar retrofleja cacuminal sonora. Ocurre en sílaba iniciada por consonante retrofleja. Por ejemplo, [pi.' tşu] 'humo'.

[n]: contoide nasal alveolar sonora. Ocurre en otros contextos. Por ejemplo, ['na.tși] 'remedio", [pə.'nən] 'pene' y [pu.'wun] 'llegué allá'.

Hay fluctuación de fonemas entre $/ \mathrm{n} / \sim / \mathrm{n} / \mathrm{y} / \mathrm{n} / \sim / \mathrm{m} /$, como se observa en los siguientes ejemplos: / wan. I en/ / wan.len్n/ 'estrella' (esta fluctuación podría asociarse con una vitalidad del fonema / n/) y / ta.kun/ / ta.kum/ 'ropa', respectivamente.

\subsubsection{Fonema $/ \mathrm{n} /$}

Este fonema se realiza como [n] : contoide nasal interdental sonora. Por ejemplo, [na.'mun] ' pie', [na.'ñø] 'nuera' y ['pun] ' noche'. Tal como se aprecia en los enunciados anteriores y en los de 4.2.12, [n] y [n] se presentan en posición inicial, entre vocales y en coda silábica, por lo que existe un contraste por pares submínimos entre estos fonos. Por lo mismo, se justifica la asignación de estatus fonémico a ambos segmentos.

Al igual que en el caso de [t] , otra razón que justifica el estatus fonémico de [n] es su aparición consistente en determinadas palabras. Así, las palabras [na.'ñøy] 'nuera', [po.'nnon] 'hígado', [ña.'muñ] 'pie', ['puñ] 'noche', [kje.'wunn] 'lengua' y [ann.'țə] 'sol' son, en su mayoría, realizadas con el fono nasal interdental. Por otra parte, siete de los informantes presentan consistentemente la realización interdental de este fonema. Valga señalar que este es el segmento interdental más realizado por los entrevistados.

Hay fluctuación de fonemas entre $/ \mathrm{n} / \sim / \mathrm{n} / \mathrm{y} / \mathrm{n} / \sim / \mathrm{m} /$, como se observa en los siguientes ejemplos: / tonn/ /tən/ 'piojo' (este caso también podría interpretarse como una influencia del español en el mapudungun de la zona) y /po.n్non/ /po.nom/ 'hígado', respectivamente. 


\subsubsection{Fonema / $\mathrm{n} /$}

Este fonema se realiza como [n]: contoide nasal mediopalatal sonora. Por ejemplo, [nai..'kii] 'gato', [ku. ni.'val] 'huérfano' y [ma.'kun] 'manta'. Hay fluctuación de fonemas entre $/ \mathrm{n} / \sim / \mathrm{n} /, / \mathrm{n} / \sim / \mathrm{n} / \mathrm{y} / \mathrm{n} / \sim /$ ठ/, como se observa en los siguientes ejemplos: /ma.kun/ /ma.kun/ 'manta' (estos casos también podría interpretarse como influencia del español en el mapudungun de la zona), / moK.vəəh/ / noK.vənn/ 'sangre', y / tjan .we/ /tjað.we/ 'chancho', respectivamente.

\subsubsection{Fonema $/ \mathrm{n} /$}

Este fonema tiene dos realizaciones alofónicas en distribución complementaria:

[n]': contoide nasal postpalatal sonora. Ocurre ante vocal anterior. Por ejemplo, ['nje] ‘ojos' y [wa.nje.'Ien] 'estrella'.

[ๆ]: contoide nasal velar sonora. Ocurre ante vocal no anterior. Por ejemplo, ['yu. fu] 'zorro', [wa.yu.'len] 'estrella', y [tşur.' tsuin] 'fruto'.

Hay fluctuación de fonemas entre $/ \mathrm{n} / \sim / \mathrm{m} /, / \mathrm{n} / \sim / \mathrm{n} /, / \mathrm{h} / \sim / \mathrm{n}$ $/ \mathrm{y} / \mathrm{y} / \sim / \mathrm{j} /$, como se observa en los siguientes ejemplos: /li.pan $/ \sim$ / li.pam/ 'brazo', /li.pay/ /li.pan/ 'brazo' (esta fluctuación también podría interpretarse como una influencia del español en el mapudungun de la zona), / ya.pin/ / pa.pin/ 'nuera', / ge/ / ne/ 'ojos' y /lam. ঘen/ / la.mjen/ 'hermana', respectivamente.

\subsubsection{Fonema /1/}

Este fonema tiene dos realizaciones alofónicas en distribución complementaria:

[l]: contoide lateral alveolar retrofleja cacuminal sonora.

Ocurre en el postmargen de la sílaba iniciada por consonante retrofleja. Por ejemplo, [ku.'tşal] 'fuego'.

[1]: contoide lateral alveolar sonora. Ocurre en otros contextos.

Por ejemplo, ['la] ‘negación', [ku.'la] 'tres' y [ka.'lal] ‘hongo'.

Hay fluctuación de fonemas entre $/ 1 / \sim /[/ \mathrm{y} / 1 / \sim / \mathrm{j} /$, como se observa en los siguientes ejemplos: / ku.ni.val/ / ku.ni.val / 'huérfano' 
(esta fluctuación podría asociarse con una vitalidad del fonema / I /) y / leư.vu/ /jeư.vu/ 'río', respectivamente.

\subsubsection{Fonema / I/}

Este fonema se realiza como [!]]: contoide lateral interdental sonora. Por ejemplo, ['lla] 'muerto', [pə.'Iə] 'mosca', y ['țol] 'frente'.

De acuerdo con el análisis fonémico, existe un contraste por pares mínimos entre [1] y [!] , como es el caso en [']la] 'muerto' y ['la] 'negación'. Además, se observa un contraste por pares submínimos, pues ambos segmentos se presentan en posición inicial, entre vocales y en coda silábica. Por otra parte, y al igual que en el caso de [t] y [n] ], otra razón que justifica el estatus fonémico de [I] es la alta consistencia en la producción de este fono. Así, por ejemplo, las palabras [pə.'İə] 'mosca' y [!la.'wen] 'remedio', entre otras, se realizan, en su mayoría, con el fono interdental.

Hay fluctuación de fonemas entre / I/ /1/, como se observa en el siguiente ejemplo: / ta.pəI/ /ta.pəl/ 'hoja'. Esta fluctuación, tal como para el caso de $/ \mathrm{t} / \sim / \mathrm{t} / \mathrm{y} / \mathrm{n} / \sim / \mathrm{n} /$, podría interpretarse como una influencia del español en el mapudungun de la zona.

\subsubsection{Fonema $/ \Lambda /$}

Este fonema se realiza como $[\Lambda]$ : contoide lateral mediopalatal sonora. Por ejemplo, ['Ka.Ka] 'suegra', [ka.'tti.Ka] 'trigo' y [ma.'məK] 'madera'. También se observan algunos casos de transferencias del español: una consonante africada alveopalatal sonora [dz] y una consonante africada palatal sonora [dj]. La primera, fue articulada en tres oportunidades en la palabra 'suegra', mientras que la segunda fue articulada en una oportunidad en la misma palabra. Así, en donde se esperaba [' $\mathrm{a} . \Lambda$ a], se articuló ['dza.dza] o ['dja.ja]. Cabe señalar que el porcentaje de apariciones de estas transferencias es baja, como se observa en el siguiente gráfico. 
Gráfico 9: Porcentaje de realizaciones alofónicas y transferencias en el fonema $/ \Lambda /$.

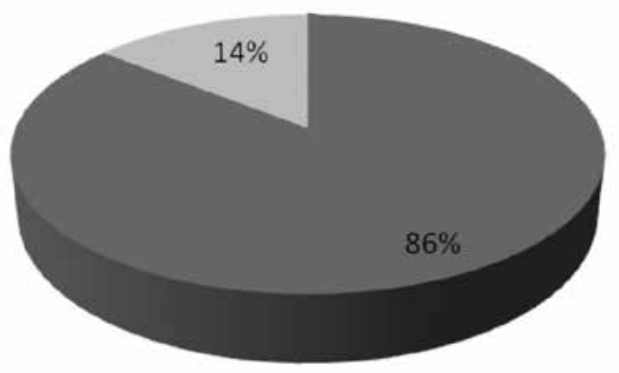

\section{- Alófonos de / $\mathrm{N} \quad$ III Transferencias}

Hay fluctuación de fonemas entre $/ K / \sim / \mathrm{j} /, / K / \sim / 1 /$ y $/ K / \sim /$ I /, como se observa en los siguientes ejemplos: / ka.tfi.Ka/ / ka.tji.ja/ 'trigo', / a.tja.waK/ /a.tja.wal/ 'pollo' (estos dos casos de fluctuación podrían interpretarse como casos de influencia del español en la lengua vernácula) y / ma.məK / / ma.məl / 'leña'. Las siguientes tablas resumen los fonos y fonemas consonánticos de la zona. 
Tabla 3: Fonos contoides de Lonquimay

\begin{tabular}{|c|c|c|c|c|c|c|c|c|c|c|c|c|c|}
\hline \multirow[b]{2}{*}{ 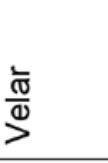 } & 㝘 & & & & & $\bar{x}$ & & 3 & 刃 & 至 & & & \\
\hline & $\begin{array}{l}\text { Dे } \\
\text { c }\end{array}$ & $\underline{z}$ & & & & & & & & & & & \\
\hline \multirow{2}{*}{ 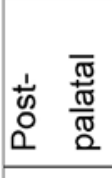 } & 亡ั. & & & & & & & & & 至 & & & \\
\hline & 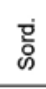 & $\overline{\underline{z}}$ & & & & & & & & & & & \\
\hline \multirow{2}{*}{ 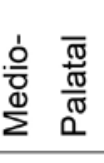 } & 宮 & & & & & 要 & & 3 & & 5 & & $\Sigma$ & \\
\hline & $\begin{array}{l}\text { 悹 } \\
\text { के }\end{array}$ & & & & & & & & & & & & \\
\hline \multirow{2}{*}{ 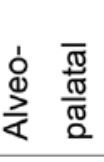 } & 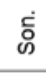 & & & & & & & & & & & & \\
\hline & 总 & & & 至? & $\underline{\underline{E}}$ & 5 & & & & & & & \\
\hline \multirow{2}{*}{ 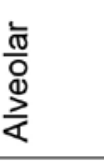 } & 㝘 & & & & & & $\mathbb{N}$ & & 3 & $\Xi$ & ㄹ & $\Xi$ & ə \\
\hline & 密 & $\Xi$ & $\Xi$ & & & $\underline{\Xi}$ & & & & & & & \\
\hline \multirow{2}{*}{ 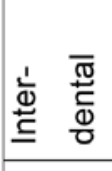 } & 灾 & & & & & 2 & & & & 도 & & $\exists$ & \\
\hline & $\begin{array}{l}\text { 嵩 } \\
\text { ஸे }\end{array}$ & $\Xi$ & & & & $\Phi$ & & & & & & & \\
\hline \multirow{2}{*}{ 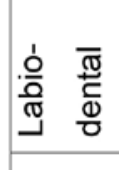 } & $\begin{array}{l}\overline{5} \\
\text { के }\end{array}$ & & & & & $\geq$ & & & & & & & \\
\hline & 宮 & & & & & $\Xi$ & & & & & & & \\
\hline \multirow{4}{*}{$\frac{\bar{\sigma}}{\overline{0}}$} & 离 & & & & & 픈 & & & & $\underline{\Xi}$ & & & \\
\hline & 总 & $\Xi$ & & & & & & & & & & & \\
\hline & & 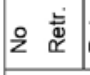 & 这 & 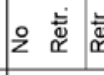 & 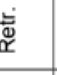 & \begin{tabular}{ll}
\multirow{2}{*}{} \\
\hdashline
\end{tabular} & 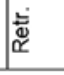 & 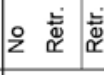 & & 竞竞 & 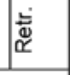 & 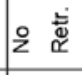 & 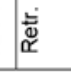 \\
\hline & & $\frac{v}{\bar{c}}$ & & 湜 & & & ¿ & 齐 & & & $\begin{array}{l}\underbrace{2} \\
z\end{array}$ & & ホேं \\
\hline
\end{tabular}


Tabla 4: Fonemas consonánticos de Lonquimay

\begin{tabular}{|c|c|c|c|c|c|c|c|}
\hline & Labial & Interdental & Alveolar & Alveopalatal & Palatal & Velar \\
\hline \multicolumn{2}{|l|}{ Oclusiva } & $/ p /$ & $/ \mathrm{t} /$ & $/ \mathrm{t} /$ & & & $/ \mathrm{k} /$ \\
\hline \multirow[b]{2}{*}{ Africada } & Retrofleja & & & & $/ \mathrm{ts} /$ & & \\
\hline & $\begin{array}{l}\text { No } \\
\text { retrofleja }\end{array}$ & & & & $/ \mathrm{t} / \mathrm{I}$ & & \\
\hline \multirow[b]{2}{*}{ Fricativa } & Plana & $/ \mathrm{v} /$ & $10 /$ & & & & \\
\hline & Cóncava & & & $/ \mathrm{s} /$ & & & \\
\hline Aproximante & Retrofleja & & & 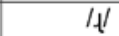 & & & \\
\hline \multicolumn{2}{|l|}{ Nasal } & $/ \mathrm{m} /$ & $\ln /$ & $\mathrm{ln} /$ & & $/ \mathrm{n} /$ & $/ \mathrm{n} /$ \\
\hline \multicolumn{2}{|l|}{ Lateral } & & II & /II & & IN & \\
\hline
\end{tabular}

\subsection{Semiconsonantes}

\subsubsection{Fonema / j/}

Este fonema tiene tres realizaciones alofónicas en variación libre y en distribución complementaria:

Aproximante palatal sonora [j]. Ocurre cuando se inicia el premargen silábico, en variación libre con [j]. Por ejemplo, ['ju] ['ju] 'nariz', [ku.'jəm] [ku.' jəm] 'arena'.

Fricativa palatal sonora [j].

Vocoide muy alta anterior no redondeada [i]. Ocurre en el postmargen silábico. Por ejemplo, ['mai] ]'sí'.

Según los datos obtenidos, [j] posee una mayor frecuencia que [j], lo que justifica su selección como forma base del fonema. Así lo muestra el siguiente gráfico.

Gráfico 10: Porcentaje de realización de los segmentos [j] y [j].

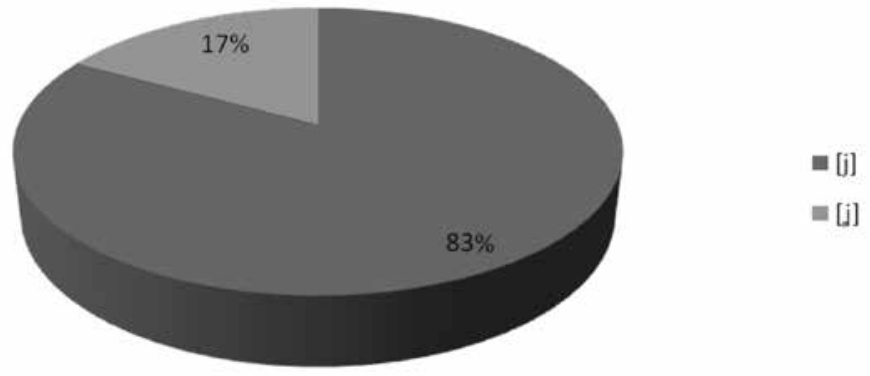


Hay fluctuación de fonemas entre $/ \mathrm{j} / \sim / 1 /$, como se observa en el siguiente ejemplo: /ja.vəj/ /ja.vəl/ 'duro'.

\subsubsection{Fonema / w /}

Este fonema tiene dos realizaciones alofónicas en distribución complementaria:

[w]: aproximante velar sonora redondeada. Ocurre en el premargen silábico. Por ejemplo, [wen.'tşu] 'hombre' y [la.'wen] 'remedio'.

[u్ ]: vocoide muy alta posterior redondeada. Ocurre en el postmargen silábico. Por ejemplo, [ku.'ðau ] 'trabajo'.

Hay fluctuación de fonemas entre /w/ /o/, como se observa en el siguiente ejemplo: / pu.wun.i.jew/ / pu.wun.i.jeo/ 'llegué allá'

\subsubsection{Fonema $/ \gamma /$}

Este fonema tiene dos alófonos en distribución complementaria:

[४]: fricativa velar sonora no redondeada. Ocurre en el

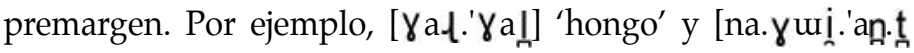
ə] 'atardecer'.

[w] ]: vocoide muy alta posterior no redondeada. Ocurre en el postmargen. Por ejemplo, [mu.' jeu్ ] 'greda'.

Hay fluctuación de fonemas entre $/ \gamma / \sim / \mathrm{j} /$, como se observa en el siguiente ejemplo: / fey.le/ / fej.le/ 'siete'.

El siguiente cuadro resume los fonemas semiconsonánticos de la zona.

Tabla 5: Fonemas semiconsonánticos de Lonquimay

\begin{tabular}{|ccc|}
\hline Oblonga & Redondeada \\
\hline$/ \mathrm{j} /$ & $/ \mathrm{\gamma} /$ & $/ \mathrm{w} /$ \\
\hline Anterior & & Posterior \\
\hline & & \\
\hline
\end{tabular}




\subsection{Fonotaxis}

\subsubsection{Estructura silábica}

Las posibilidades silábicas del mapudungun hablado en Lonquimay son las siguientes:

\begin{tabular}{|c|c|c|}
\hline V & /i.lo/ & 'carne' \\
\hline $\mathrm{CV}$ & /u.le/ & 'mañana' \\
\hline $\mathrm{ScV}$ & /ja.vəj/ & 'duro' \\
\hline VC & /an.t.tə/ & 'sol' \\
\hline VSc & /əj/ & 'nombre' \\
\hline CVC & /moর.vəر/ & 'sangre' \\
\hline CVSc & /ja.vəj/ & 'duro' \\
\hline ScVC & /wen.tsu/ & 'hombre' \\
\hline ScVSc & /u.jew/ & 'greda' \\
\hline
\end{tabular}

Premargen silábico

En el premargen silábico ocurren todas las consonantes, como se observa en los siguientes ejemplos:

\section{Oclusivas}

/p/: /pa.jun/ 'bigote' /t/: /to.ki/ 'hacha /k/: /ko/ 'agua'

/țt: /țan/ 'piojo'

\section{Nasales}

$/ \mathrm{m} /:$ /mə.ța/ 'cuerno' /n/: /na.tsil 'natre' /n/: /na.mun/'pie' /n/: /nu.ke/ ' madre'

/n/: /na.pin/ 'recién casada'

\section{Fricativas}

/v/: /va.tfan.tə/ 'hoy'

/s/: /san.we/ 'chancho'

/ð/: /ða.ne/ 'nido'

\section{Africadas}

/ts/: /tsal.kan/ 'trueno'

/tf/: /tfa.đi// 'sal'

\section{Laterales}

/l/: /la.wen/ 'remedio'

IIV: /laj/ 'morir'

IN: INa.Kal 'suegra'

\section{Aproximante}

/ l/: / |ə..jo/ 'harina cruda' 
Semiconsonantes

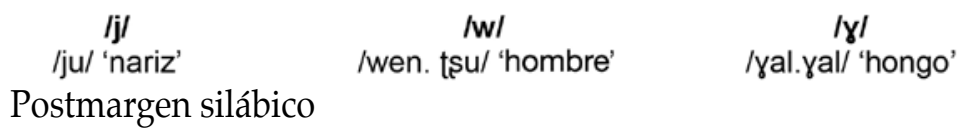

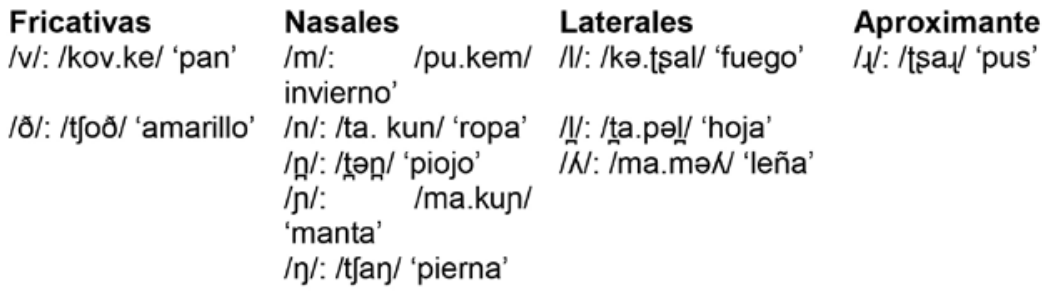

Semiconsonantes

$\begin{array}{ccc}\text { /j/ } & / \mathrm{w} / & / \mathrm{\gamma} / \\ \text { /naj.ki/ 'gato' } & \text { /kə.ðaw/ 'trabajo' } & \text { / ley.le/ 'siete' }\end{array}$

\subsubsection{Estructura de la palabra}

Las palabras del mapudungun hablado en Lonquimay pueden tener una, dos o tres sílabas:

\begin{tabular}{|l|l|l|}
\hline Monosilábicas & Bisilábicas & Trisilábicas \\
\hline /ju/ 'nariz' & /ann.ț// 'sol' & /ku.nu.val// 'huérfano' \\
\hline
\end{tabular}

\section{Comparación con otra variedad cordillerana}

A continuación se realizará una comparación entre la variante que nos ocupa y el chedungun hablado en las comunidades de Trapa-Trapa y Butalelbun, ambas pertenecientes a la zona de Alto Bío-Bío. La descripción fonético-fonológica de la variante hablada en estas localidades fue realizada por Manuel Jiménez, en enero de 2014, bajo el formato de tesis para optar al grado de Licenciado en Educación con mención en Español. Dicho estudio se inserta en el marco del proyecto Fondecyt № 1131095.

5.1. Forma básica de los fonemas fricativos planos

a) Para el fonema fricativo plano labiodental 
Gráfico 11: Comparación de las realizaciones del fonema fricativo plano labiodental en 2 localidades de Alto Bí-Bío y en Lonquimay.

Trapa Trapa / Butalelbun

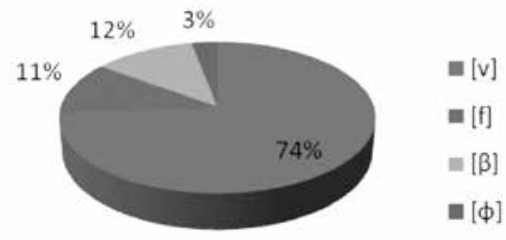

Lonquimay

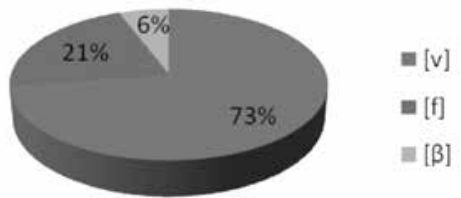

Como se observa, en ambas zonas existe una alta ocurrencia del fono [v], lo que lleva a seleccionarlo como la forma base del fonema en ambos sectores. Asimismo, ocurren los fonos planos labiodental sordo [f] y bilabial sonoro [ $\beta$ ], con una leve diferencia en los porcentajes de ocurrencia: el fono [f] ocurre más en Lonquimay (21\%) que en Alto BíoBío (11\%), y el fono fricativo bilabial sonoro dobla su ocurrencia en Alto Bío-Bío (12\%). Ahora, no obstante las similitudes en ambas zonas, se observa la presencia del fono fricativo bilabial sordo [ $\phi]$ en Alto Bío-Bío, con un 3\% de ocurrencia, mientras que en Lonquimay éste no ocurre, según los datos recogidos en nuestra investigación.

\section{b) Para el fonema fricativo plano interdental}

Gráfico 12: Comparación de las realizaciones del fonema fricativo plano interdental en 2 localidades de Alto Bio-Bío y en Lonquimay.
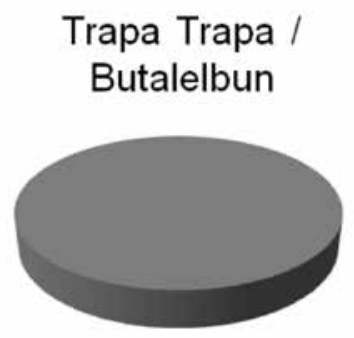

\section{Lonquimay}

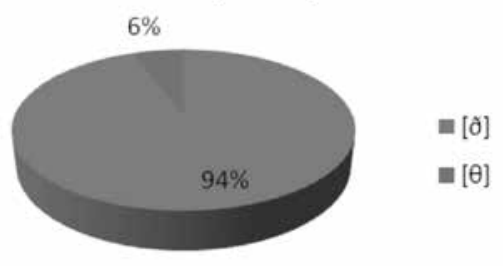


Como se observa, en el chedungun hablado en las dos localidades de Alto Bío-Bío, la realización fricativa plana interdental sonora [ð] ocurre en el $100 \%$ de los casos, convirtiéndose en la única realización de este fonema; mientras que en el mapudungun hablado en Lonquimay, aunque escasas, también ocurren las realizaciones interdentales sordas [ $\theta$ ]. Ahora bien, no obstante esta diferencia, en cada sector se justifica seleccionar el fono [ð] como la forma básica del fonema (/ð/).

\subsection{Vigencia de las interdentales}

Gráfico 13: Porcentajes de realizaciones interdentales y alveolares de los fonemas $/ \mathbf{t} /, / \mathrm{n} /$ y / $/$ en dos localidades de Alto Bío-Bío.

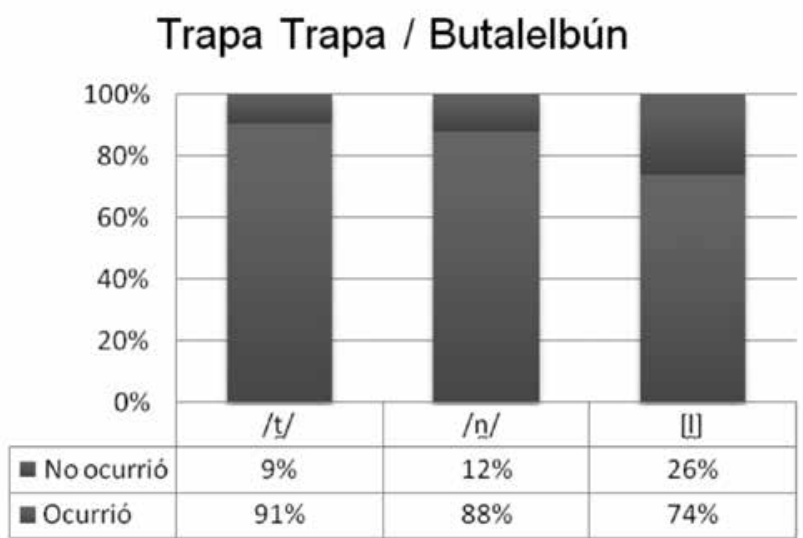

Gráfico 14: Porcentajes de realizaciones interdentales y alveolares de los fonemas $/ \mathrm{t} /, \mathrm{n} / \mathrm{n} / \mathrm{I} / \mathrm{en}$ Lonquimay.

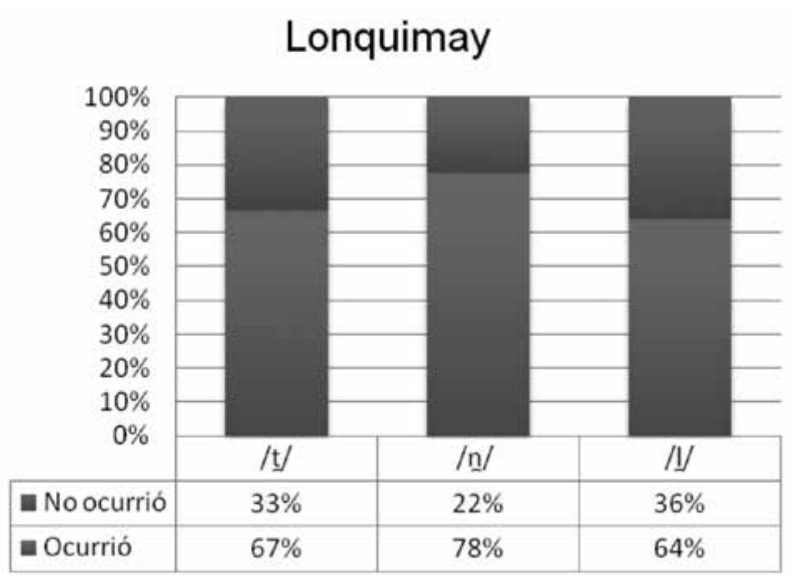


De acuerdo con los gráficos precedentes, en ambas zonas se presenta una alta ocurrencia de los segmentos interdentales, en las palabras donde era expectable su aparición. Ahora, la mayor frecuencia en Alto Bío-Bío podría explicarse por su aislamiento geográfico. Sin embargo, aun cuando en ambas zonas la lengua vernácula está en contacto con el español, estos segmentos mantienen una importante vitalidad.

\subsection{Alofonía de / ts /}

Gráfico 15: Porcentajes de realizaciones del fonema /tss/ en dos localidades de Alto Bío-Bío y en Lonquimay.

Trapa Trapa /

Butalelbun

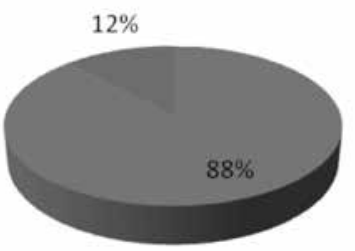

Lonquimay

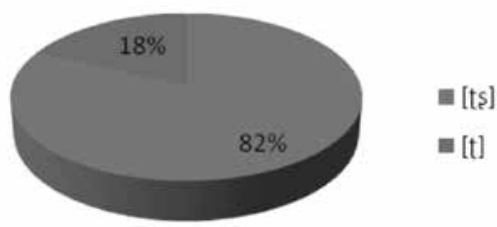

Como se observa, en ambas zonas existen dos realizaciones alofónicas del fonema / ts / y ambos segmentos ocurren con porcentajes similares: más de un $80 \%$ para [ts] y menos de un $20 \%$ para [t]; por lo mismo, conviene escoger el fono [ts] como la forma básica del fonema.

\subsection{Alofonía de / $1 /$}

Gráfico 16: Porcentajes de realizaciones del fonema / $\mathbf{f}$ en dos localidades de Alto Bio-Bí y en Lonquimay.

\section{Trapa Trapa /Butalelbun}

\section{Lonquimay}
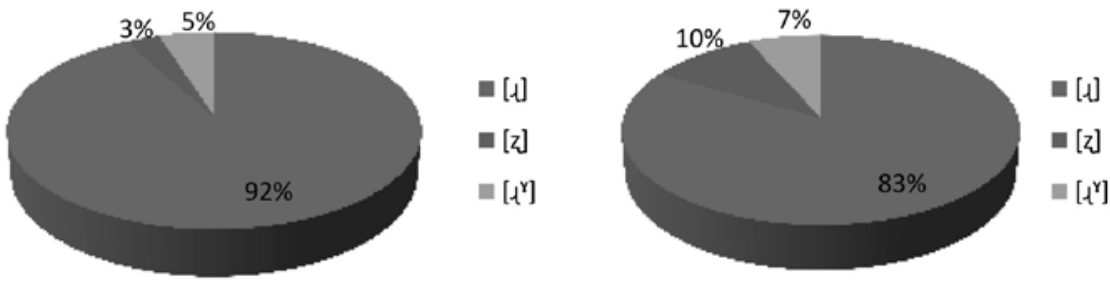
Como se observa, en ambas zonas se presentan tres realizaciones alofónicas del fonema / $\downarrow /:[\iota],[z]$ y $\left[\iota^{\gamma}\right]$. La mayor ocurrencia de $[\iota](92 \%$ en Trapa Trapa/ Butalelbun y 83\% en Lonquimay) justifica su selección como la forma base del fonema. La realización aproximante retrofleja velarizada sonora $\left[f^{\gamma}\right]$ también presenta un porcentaje de aparición similar: $5 \%$ en Alto Bío-Bío y 7\% en Lonquimay; mientras que la realización fricativa retrofleja sonora $[z]$ posee una ocurrencia un poco mayor en Lonquimay (10\%) que en Alto Bío-Bío (3\%).

\subsection{Transferencias del español}

Gráfico 17: Porcentajes de realizaciones vernáculas y transferencias en los fonemas / $/ \mathbf{\$} /, / v /, / \circlearrowright /$, y lj/ en dos localidades de Alto Bio-Bio.

\section{Trapa Trapa / Butalelbun}

\begin{tabular}{|c|c|c|c|c|}
\hline \multicolumn{5}{|l|}{$100 \%$} \\
\hline & & & & \\
\hline & & & & \\
\hline $60 \%$ & & & & \\
\hline $40 \%$ & & & & \\
\hline $20 \%$ & & & & \\
\hline $0 \%$ & [ts] & [v] & [j] & {$[\tilde{0}]$} \\
\hline - Fonos vernáculos & $99 \%$ & $98 \%$ & $98 \%$ & $83 \%$ \\
\hline - Transferencias & $1 \%$ & $2 \%$ & $2 \%$ & $17 \%$ \\
\hline
\end{tabular}

Gráfico 18: Porcentajes de realizaciones vernáculas y transferencias en los fonemas $/ \mathrm{t} \mathbf{s} /, / \mathrm{v} /, / \mathrm{d} /$, $y / \widehat{K} /$ en Lonquimay.

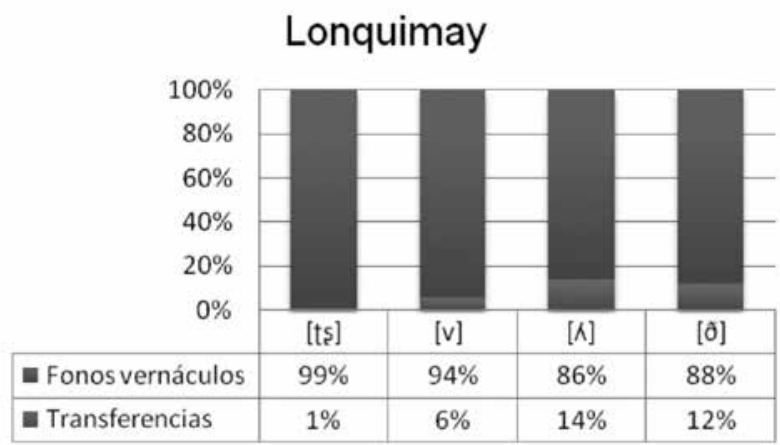


Como se observa, en ambas zonas las transferencias son escasas o mínimas. Así, en Alto Bío-Bío, el caso de transferencia más alta es para /ð/, con un $17 \%$, mientras que para /v/ y /j/, un $2 \%$, y para / ts /, sólo es del $1 \%$. En el caso de Lonquimay, ocurre algo similar: para $/ \Lambda /$, el porcentaje es del $1 \%$; para /v/, de un $6 \%$; para /ð/, de un $12 \%$, mientras que para $/ K /$, de un $14 \%$, con lo que se constituye en el porcentaje más alto.

\section{Conclusiones}

Presentamos aquí un resumen de los aspectos más prominentes de la fonología de la zona estudiada, en tanto focos de discusión centrales en el estudio del nivel fónico del mapudungun.

\section{Sonoridad de las fricativas}

Como hemos visto, en el mapudungun hablado de Lonquimay existe una manifiesta tendencia a la sonoridad de las consonantes fricativas labiodental e interdental, característica que se asemeja al mapudungun hablado en Tirúa y Alto Bío-Bío, ya que en ambas zonas predominan tales realizaciones.

Como se observa en 4.2.8., el fonema fricativo plano interdental / / se realiza, mayoritariamente, sonoro. Decimos "mayoritariamente", porque hubo 6 realizaciones interdentales áfonas $[\theta]$, en variación libre con [ð], en coda silábica, como es el caso en [pa.'nuð] [pa.'nu $\theta$ ] 'suave',

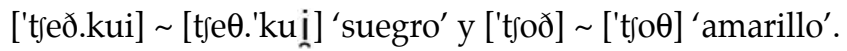

Una situación similar ocurre con el fonema fricativo labiodental, donde además de la variante labiodental sonora, en ocasiones se presentan los alófonos [f] y [ $\beta]$, cuyos porcentajes de aparición se muestran en 4.2.7. Según nuestros datos, el fono fricativo plano labiodental sordo [f] ocurre sólo en coda silábica. Así, palabras como 'viento' y 'pan' se pronuncian en su mayoría [ku.'‘uv] y ['kov.kje], respectivamente, sin embargo, en algunos casos, alternan con ['ku. „uf] y ['kof.kje]. Por su parte, [ $\beta]$ alterna libremente con $[\mathrm{v}]$ en el premargen silábico y entre vocales, como es el

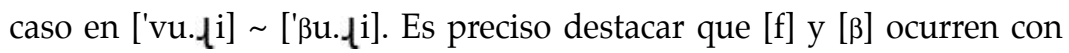
una frecuencia significativamente menor que [v]. Los siguientes gráficos muestran los porcentajes de realizaciones sonoras y áfonas para cada fonema. 
Gráfico 19: Porcentajes de realizaciones sonoras y áfonas de /v/.

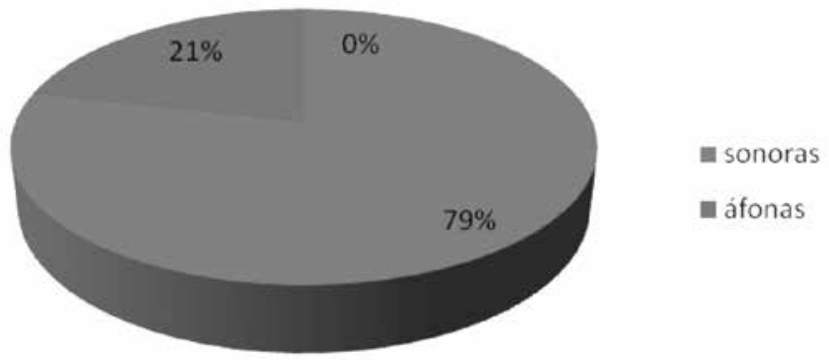

Gráfico 20: Porcentajes de realizaciones sonoras y áfonas de/ð/.

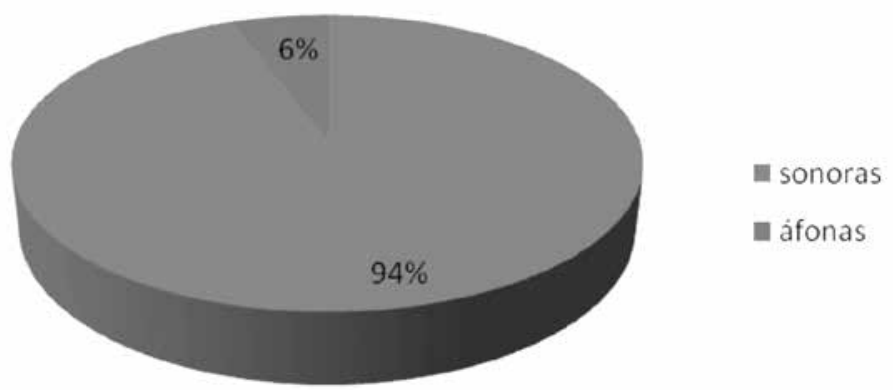

Estos resultados se aproximan a los que exhiben las variantes septentrionales, descritas por Sánchez (1989), Salamanca (1997), Salamanca y Quintrileo (2009), Jiménez (2014) y Toro (2014); y se distancian de aquellos que muestran las variantes de la zona centro y sur de la Araucanía, descritas por Echeverría (1964), Salas (1976), Lagos (1981), Álvarez-Santullano (1986) y Sadowsky et al. (2013).

\section{El estatus fonémico de las interdentales}

Como se observó en la descripción de los fonemas consonánticos, los fonos interdentales [t] $]$ [n] y [ [I] poseen estatus fonémico en el mapudungun hablado en Lonquimay. Así lo demuestran los siguientes pares submínimos: 
[t]

$\#$

V_V [nji.Na. 'tun] 'rogativa'

[n]

\#

V_V [pə.'nən] 'pene'

_\# [pu. 'wun] 'llegué allá'

[l]

\#__ [lam. 'njen] 'hermana'

V_V ['km.la] 'tres'

_ \# [ka.' lal] 'hongo' [t]

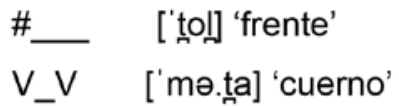

[n]

\#__[na. 'mun] 'pie'

V_V [na. 'nəฑ] 'nuera'

\# ['pun] 'noche'

[1]

\#

[la. 'wen] 'remedio'

V_V ['pə.lə] 'mosca'

\# [ta.'pel] 'hoja'

Otra razón que justifica su estatus fonémico es la alta consistencia en la realización de los fonos interdentales, tanto en las palabras elicitadas, como en los colaboradores. Así, las palabras expectables de realización con consonantes interdentales fueron producidas en su mayoría con dichos fonos, como es el caso en ['toll] 'frente', ['pu n] 'noche', ['mə.ta] 'cuerno', entre otras. Por otra parte, algunos de nuestros colaboradores muestran una alta consistencia en la realización de los fonos interdentales a lo largo de toda la lista léxica. Tal es el caso de Lilian Puel, Berta Curical, Arturo Lillo y Elena Rosas. Esta última, además, mostró una alta conciencia lingüística, al explicar la diferencia en la pronunciación de 'provocar vómito' (/ Iolon/) y 'hacer un hoyo en la tierra' (/lolon/).

En este sentido, coincidimos con Echeverría (1964), Salas (1976), Lagos (1981), Salamanca (1997) y Sadowsky et al (2013) en cuanto a la asignación de estatus fonémico a la serie interdental, y nos distanciamos de los postulados de quienes destacan la ausencia de interdentales fonémicas (Sánchez, 1989) o, incluso, que éstas están prácticamente perdidas en tanto fonos (Croese, 1980). En nuestra experiencia, la serie interdental, si bien no ostenta la vitalidad que poseía otrora, sí forma parte del inventario fonémico en el mapudungun hablado en Lonquimay por las razones y datos ya expuestos. 


\section{El fono [ []}

Como se observa en 4.2.6., el fonema / $\mathrm{t} /$ presenta dos realizaciones alofónicas en variación libre. Una de esas realizaciones corresponde al fono fricativo alveopalatal áfono [s], cuya ocurrencia sólo se detectó en posición intervocálica, como es el caso en [ka.'tfi.Ka] [ka.'tfi.ja] 'trigo' y

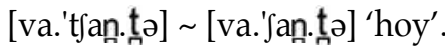

En este contexto, nuestros resultados se avienen con los presentados por Echeverría (1964) y Salamanca \& Quintrileo (2009); y se distancian de los presentados para la variante de Alto Bío-Bío (Sánchez, 1989 y Salamanca, 1997), donde el fono [f] no se presenta. Estos resultados tampoco coinciden con los que se presentan en Salas (1976) y Sadowsky et al. (2013), donde este fono es interpretado como un alófono de / s / y como un fonema distinto $/ \mathrm{s} /$, respectivamente.

\section{La presencia del fono "aproximante velarizado" del fonema aproximante retroflejo}

Como se señaló en 4.2.10, el fonema retroflejo sonoro / $/$ / tiene entre sus variantes un fono velarizado $\left[f^{\gamma}\right]$. Dicho fono no es reportado en las descripciones tradicionales del mapudungun hablado en Chile. Una excepción en este punto la constituye el trabajo de Henríquez y Salamanca (2012), en donde se señala como uno de los rasgos prominentes de la fonología del chedungun hablado por escolares del Alto Bío-Bío la presencia de un fono velar aproximante, que representan [§ฺ]. Explícitamente señalan:

Otro aspecto destacable es la ocurrencia en Butalelbun de un fono velar aproximante [§ฺ], el cual, al menos con esta descripción y notación, no ha sido resportado en el habla adulta de la Araucanía en general. Hay diferencias articulatorias y funcionales entre

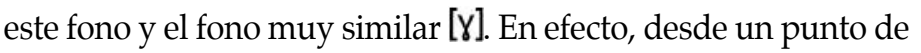
vista articulatorio, se distinguen en que mientras $[\gamma]$ es un fono plenamente fricativo y velar, y se articula con la lengua en una posición neutra ("en reposo"), [ð] ] es aproximante y la posición de la lengua muestra una leve retroflexión, atribuible, a nuestro juicio, al estadio anterior fricativo retroflejo del cual parece ser una evolución. Desde un punto de vista funcional, en tanto, difieren en que mientras $[\gamma]$ es un alófono de $/ \gamma /$ y siempre se adelanta ante vocales anteriores (así ocurre, por ejemplo, 
en la palabra [ðе' $\left.\left.\gamma^{j i n}\right]\right),[\gamma]$ es un alófono de / ^/ y no suele adelantarse ante vocales anteriores, de modo que una palabra como "espalda" (/vuцi/) se pronuncia habitualmente [vu' y no [vu' $\left.\gamma^{j i}\right]$ ) (Henríquez y Salamanca, 2012: 165).

Coincidimos con esta exhaustiva descripción. Sin embargo, preferimos utilizar el símbolo que se contiene en Jiménez (2014) y Toro (2014), esto es $\left[f^{\gamma}\right]$, pues creemos que, al incorporarse el rasgo de retroflexión, se da cuenta de manera aún más fina de la naturaleza articulatoria de su producción.

\section{Transferencias fonológicas o casos de castellanización del mapudungun}

Las transferencias fonológicas son fenómenos característicos en situaciones de lenguas en contacto. Así, en el caso del mapudungun hablado en Lonquimay, se observan algunas transferencias fonológicas desde el castellano al mapudungun. Por ejemplo, en algunos casos donde era expectable el fono fricativo plano interdental [ð], hubo realizaciones con el fono oclusivo postdental sonoro [d] (como en la palabra 'mujer', la cual fue realizada como [do.'mo.tje] y no como [ðo.'mo.tje]). Asimismo, en algunos casos donde se esperaba el fono fricativo plano labiodental sonoro [v], se presentó la realización bilabial oclusiva [b], como es el caso en 'espalda', cuya realización fue [bu.zi], en vez de [vu.zii]. Otro caso de transferencia se presenta en 'remedio desagradable', donde un informante reemplaza el fono africado alveolar retroflejo áfono [ts] por la secuencia [tr] (donde se esperaba ['na.tsi], se articuló ['na.tri]). Por último, ocurren también dos realizaciones del fonema $/ K /$ atribuibles al contacto: $[d]$ ] y [dj]. Ahora, aunque estas transferencias no son cuantitativamente prominentes, tal como se observa en 5.5, sí se presentan en un porcentaje ligeramente mayor que en el Alto Bío-Bío, lo que puede revelar un proceso de castellanización un tanto más pronunciado.

\section{Estatus fonémico del fono [ $\mathrm{\gamma}]$}

Como se observa en 4.3.3, el fono [ $\mathrm{\gamma}$ ] tiene estatus fonémico en Lonquimay $(/ \gamma /)$ y presenta dos realizaciones alofónicas $([\gamma]$ y $[w])$ en distribución complementaria. El fono [४] ocurre en el premargen, mientras que $[\underset{n}{w}]$ ocurre en el postmargen. 
En este contexto, nuestros resultados se avienen con los presentados, entre otros autores, por Salas (1976) y Salamanca (1997); y se distancian de la interpretación de Echeverría (1964) y Sánchez (1989), quienes no asignan estatus fonémico $\mathrm{a}[\mathrm{\gamma}$ ], pues lo consideran un apoyo consonántico de la vocal [i]. Según los datos de nuestra investigación, [y] no sólo es un desarrollo consonántico de la sexta vocal, sino que también aparece en otros contextos, como es el caso en [yal.'yal] 'hongo'. De ahí que, como hemos señalado, se le asigne estatus fonémico.

En suma, si bien existe una apreciable unidad entre todos los dialectos del mapuche, se requiere una cobertura mayor, para decantar aún más las características propias de las distintas variantes y, de este modo, hacer propuestas para que las micro-variantes reciban los materiales escritos con las características fónicas y léxicas propias y no aquellas que se presumen transversales a toda el habla mapuche. Precisamente, nuestro estudio, junto con la investigación que se lleva a cabo en el contexto del Proyecto Fondecyt $N^{\circ} 1131095$ y algunas tesis en curso que describen otras variantes cordilleranas, apuntan en esta dirección. 


\section{Referencias bibliográficas}

Álvarez-Santullano, P. (1986). Descripción fonológica del huilliche, un dialecto del mapuche o araucano del centro-sur de Chile. Tesis de Magíster en Artes con Mención en Lingüística. Universidad de Concepción.

Burquest, D. (2009). Análisis fonológico: un planteamiento funcional. Recuperado en mayo de 2013, de http:// www01.sil.org/silepubs/Pubs / 52264/Burquest_ AnalisisFonologico_52264.pdf

Croese. R. (1980). "Estudio dialectológico del mapuche". Estudios Filológicos 15, pp. 7-38.

Echeverría, M. (1964). "Descripción fonológica del mapuche actual". Boletín del Instituto de Filología de la Universidad de Chile XVI, pp. 13-59.

Henríquez, M. (2004). "Interferencias del sistema fonológico del español en el sistema fonológico de jóvenes hablantes bilingües". RLA 42(2), pp. 93-106.

(2013). Vitalidad fonológica del mapudungun en escolares mapuches pewenches y lafkenches de la VIII Región del Bío-Bío. Tesis para optar al grado de Doctor en Lingüística. Universidad de Concepción.

Henríquez, M. \& Salamanca, G. (2012). “Rasgos prominentes de la fonología segmental del chedungun hablado por escolares del Alto Bío-Bío". Alpha 34, pp. 153-171.

Jiménez, M. (2014). Fonemas segmentales y realizaciones alofónicas del chedungún hablado en las comunidades de Trapa Trapa y Butalelbun, Alto Bío-Bío. Tesis para optar al grado de Licenciado en Educación con Mención en Español. Universidad de Concepción.

Lagos, D. (1981). "El estrato fónico del mapudungu(n)". Nueva Revista del Pacífico 19-20, pp. 42-66. 
Pike, K. (1947). Phonemics. A technique for reducing languages to writing. Ann Arbor: The University of Michigan Press.

Sadowsky, S., Painequeo, H., Salamanca, G. y Avelino, H. (2013). "Mapudungun". Journal of the International Phonetic Association 43, pp. 87-96.

Salamanca, G. (1997). “Fonología del pehuenche hablado en el Alto BíoBío". RLA 35, pp. 113-124.

\& Quintrileo, E. (2009). “El mapuche hablado en Tirúa: Fonemas segmentales, fonotaxis y comparación con otras variedades". RLA 47, pp. 13-35.

Salas, A. (1976). “Esbozo fonológico del mapudungun, lengua de los mapuches o araucanos de Chile central". Estudios Filológicos 11, pp. 143-153.

(2006[1992]). El mapuche o Araucano. Fonología, gramática y antología de cuentos. Santiago: CEP.

Sánchez, G. (1989). "Relatos orales en pewenche chileno". Anales de la Universidad de Chile. Estudios en honor de Yolando Pino Saavedra 17, pp. 289- 360.

Toro, S. (2014). Descripción fonológica del chedungún hablado en los sectores de Chenqueco y Chevquelavquen, Alto Bio-Bio. Tesis para optar al grado de Licenciado en Educación con Mención en Español. Universidad de Concepción. 